\title{
Metabolic Syndrome and Risk of Colorectal Cancer: Results of Propensity Score-based Analyses in a Community-based Cohort Study
}

\author{
Jinsun Kim \\ National Cancer Center \\ Eunjung Park \\ National Cancer Center \\ Min Kyung Lim \\ National Cancer Center \\ Jin-Kyoung Oh \\ National Cancer Center

\section{Byungmi Kim} \\ National Cancer Center
}

Eun Young Park ( $\sim$ goajoa@ncc.re.kr)

National Cancer Center https://orcid.org/0000-0003-4859-6782

\section{Research article}

Keywords: metabolic syndrome, colorectal cancer, propensity score methods, cohort

Posted Date: January 16th, 2020

DOI: https://doi.org/10.21203/rs.2.21054/v1

License: () (1) This work is licensed under a Creative Commons Attribution 4.0 International License. Read Full License

Version of Record: A version of this preprint was published at International Journal of Environmental Research and Public Health on November 23rd, 2020. See the published version at https://doi.org/10.3390/ijerph17228687. 


\section{Abstract}

Background: This study aimed to determine the effects of metabolic syndrome (MetS) on colorectal cancer (CRC) using propensity score (PS) methods.

Methods: The study subjects were 2,417 men and 4,568 women from the Korean National Cancer Center (KNCC) Community Cohort between 2003 and 2010 who were followed up by 2016. We calculated adjusted hazard risks (HRs) and 95\% confidence intervals (Cls) using unadjusted and multiple Cox's hazard regression models, PS matching analysis, regression models adjusted by the PS or stratified into five strata according to PS, and PS weighting methods.

Results: In women, MetS and abnormally high triglyceride (TG) levels were associated with an increased risk of CRC (HR for MetS, 2.11 [95\% Cl, 1.21-3.86] using the multiple Cox's hazard regression and 2.19 [95\% Cl, 1.10-4.36] using the PS matching analysis; HR for abnormal TG levels, 2.09 [95\% Cl, 1.21-3.62] using the multiple Cox's hazard regression and 2.10 [95\% Cl, 1.08-4.08] using the PS matching analysis). In men, MetS did not show a significant association with the risk of incident CRC no matter which analyses we performed ( $\mathrm{HR}, 0.92[95 \% \mathrm{Cl}, 0.42,2.04]$ using the PS matching analysis).

Conclusions: Our study might provide additional evidence that deteriorated metabolic profiles increase the risk of CRC in women rather than men. Thus, it may have an important role in effective populationlevel interventions for deteriorated metabolic profiles at an early stage.

\section{Background}

Colorectal cancer (CRC) is the third most common cancer in both sexes worldwide $(1,360,602$ cases, $9.7 \%$ of the total cancer burden). According to GLOBOCAN 2018, the ranking of the estimated age-standardized rates of CRC incidence for both sexes at all cancer sites were observed to be similarly high ranked in most countries, ranking fourth in the United States, third in Europe, second in Japan, and third in the Republic of Korea [1].

Worldwide, over a billion people are known to be affected with Metabolic syndrome (MetS). The MetS prevalence are increasing in low socio-economic countries as well as high socio-economic countries. In even young adults, the prevalence ranges from 5 to $7 \%$ worldwide, although it increases with age. MetS is a complex disorder characterized by a cluster of moderate levels of metabolic, anthropometric, and hemodynamic abnormalities, accepted as a modifiable risk factor $\mathrm{CRC}$, although the mechanism linking MetS and CRC has not been clearly elucidted [2,3]. Recent systematic reviews and meta-analyses have conclusively reported that MetS is associated with an approximately 1.3-fold increased risk of CRC in both sexes, although the risk in women was slightly higher than that in men [2, 4]. A few studies have been conducted in the Republic of Korea among national health insurance subscribers or subjects who underwent colonoscopy for a health examination in a hospital. However, these studies reported inconsistent results [5-7]. So far, there are limited evidences on the association between MetS and CRC, especially for the Asian population. Furthermore, the findings of previous observational studies have 
pointes inevitably lower causality than randomized controlled trials due to selection biases. In order to overcome this weakness of observational studies, propensity score (PS)-based methods were proposed to attenuate selection biases by balancing many covariates $[8,9]$. Studies using these methods have been increasingly published in a wide range of fields, including some observational studies on MetS [10, $11]$.

In this context, this study investigated the impact of MetS on CRC incidence by conducting PS-based analyses considering age, alcohol consumption, smoking, high animal fat intake, obesity, a lack of dietary fiber intake, and a lack of physical activity etc. that have been identified as modifiable risk factors for CRC $[2,3]$, in a community-based prospective cohort in the Republic of Korea,

\section{Methods}

\section{Data source andstudy population}

The Korean National Cancer Center (KNCC) Community Cohort, as a community-based prospective cohort, was conducted by the KNCC and included 16,304 men and women who resided in Changwon-si, Chuncheon-si, Chungju-si, Sancheong-gun, and Haman-gun in the Republic of Korea [12]. The questionnaire survey was conducted by well-trained interviewers and included the following demographic information: age, sex, home region, education level, occupational history, marriage status, average household income, alcohol consumption, smoking status, physical activity, dietary intake, history of cancer, and exposure to pesticides. Additionally, the results of anthropometric measurements and clinical laboratory examinations were included. All study participants are followed through linkage to the Korean Central Cancer Registry for cancer incidence and mortality by 2016. This cohort were linked to mortality data of Statistics Korea for all participants by 2016.

After the exclusion of 9,319 participants, including those who had no history of cancer before entry, did not participate in the nutrition survey, developed cancers other than CRC, and had missing data for MetS, alcohol consumption, smoking, physical activity, diet, and education, the remaining 8,658 participants $(2,417$ men and 4,568 women) were finally eligible for the analysis (Figure 1$)$. The study was approved by the KNCC Institutional Review Board (IRB No. NCC2016-0300). All participants had provided written informed consent.

\section{Definition of CRC and MetS}

The outcome of this study was CRC incidence; the type of cancer was coded as C18, C19, and C20 according to the International Classification of Diseases 10th edition (ICD-10).

We used the National Cholesterol Education Program-Adult Treatment Panel III (NCEP-ATP III) definition of MetS as a condition with at least three of the following five risk factors concurrently: abdominal obesity (waist circumference (WC) $>102 \mathrm{~cm}$ for men and $>88 \mathrm{~cm}$ for women), hypertension (systolic blood pressure $[\mathrm{SBP}] \geq 130 \mathrm{mmHg}$ and diastolic blood pressure [DBP] $\geq 85 \mathrm{mmHg}$ ), low high-density 
lipoprotein (HDL) cholesterol level ( $<40 \mathrm{mg} / \mathrm{dL}$ for men and $<50 \mathrm{mg} / \mathrm{dL}$ for women), high triglyceride (TG) level ( $\geq 150 \mathrm{mg} / \mathrm{dL}$ ), and abnormal fasting blood sugar (FBS) level ( $\geq 110 \mathrm{mg} / \mathrm{dL}$ ) [13]. In our study, we adopted the body mass index $(\mathrm{BMI}) \geq 25 \mathrm{~kg} / \mathrm{m} 2$ as a measure of abdominal obesity due to the absence of WC data.

\section{Statistical analyses}

Descriptive analyses between subjects who developed CRC and those who did not were performed for continuous parameters using the Mann-Whitney $\mathrm{U}$ test and for categorical parameters using the Chisquare test and Fisher's exact test.

To elucidate associations between MetS and risk of CRC in both sexes and an association between abnormal TG levels and CRC in women, hazard ratios (HRs) and 95\% confidence intervals (Cls) were estimated by the following statistical analyses using Cox's hazard regression models: unadjusted and multiple regression, PS matching analysis, regression adjustment with the PS, and PS weighting methods. In these analyses, we included the following covariates known as the known risk factors of CRC $[2,14,15]$ : age, alcohol consumption (non-drinkers, moderate drinkers $[<24 \mathrm{~g} /$ day], heavy drinkers $[\geq 24$ $\mathrm{g} /$ day]), smoking status (non-smokers, moderate smokers [ $<20$ pack-year], heavy smokers [ $\geq 20$ packyear]), moderate-intensity physical activity (days/week), frequency of fruit or vegetable intake (days/week), frequency of intake of beef or pork (days/month), education level (illiterate, middle school or less, high school, and college or more), and study area (Changwon-si, Chuncheon-si, Chungju-si, Sancheong-gun, and Haman-gun).

To perform PS-based methods, we needed first to calculate the PS. The PS is the probability that an individual would have MetS or abnormal TG levels based on personal demographic and lifestyle information, and it was obtained from the fit of a Cox's hazard regression model adjusted with all the covariates mentioned above.

To evaluate the balance in baseline characteristics in the dataset used for different regression models, we calculated the standardized mean differences, and values less than 0.1 were considered negligible differences. First, we applied a 1:1 case-control matching to the PS technique, which is the eighth digit to first digit greedy matching method. This method may result in a drop-out of unmatched cases for the best matching, and thus, the number of subjects for the matching analyses was 1,038 men for MetS, 2,580 women for MetS, and 2,786 women for abnormal TG levels. Second, we performed weighted Cox's hazard regression models that were considered for an adjusted MetS effect after stratifying into 5 strata according to the quintiles of the estimated PS [16]. Additionally, a Cox's hazard regression model that used the PS as a covariate (a continuous variable and a categorical variable by quintiles) was a simple PS method similar to traditional regression analysis. Lastly, there are two major PS weighting methods referred to as standardization methods that depend on the establishment of a standard population. One is the inverse probability-of-treatment weighted (IPTW) model [17]. It considers the overall study participants as the standard population, and it uses weights of (1/PS) for those with MetS and [1/(1PS)] for those without MetS. The other is the standardized mortality ratio weighted (SMRW) model. It 
regards those without MetS as the standard population and applies weights of 1 for those with MetS and [PS/(1-PS)] for those without MetS.

Statistical analyses were carried out using SAS software, version 9.3 (SAS Institute, Cary, NC, USA) and R version 3.4.3 using the 'tableone' package [18] and were two-sided, with a significance level of $p<0.05$.

\section{Results}

In total, 2,417 male and 4,568 female participants were included in this study (Figure 1). Table 1 shows the baseline characteristics of the study population. Among the study subjects, there were 57 men and 54 women newly diagnosed CRC after the entry of this cohort. The mean age of the study subjects was approximately 60 years old (data not shown), which is older than the general Korean population. CRC cases were older than non-cancer cases. Male participants had a high percentage of smokers and drinkers, whereas most of the female participants were non-smokers or non-drinkers. Five hundred twenty-four men (21.7\%) and 1,297 (28.4\%) women had at least three components of MetS at the entry of a cohort and CRC cases in both sexes were significantly more likely to have MetS, and those in women might have higher TG levels.

Tables S1 and Figure S1 show male participants' characteristics according to the presence of MetS as well as the degree of imbalance among covariates. Also, Tables S2 and S3 and Figure S2 show female participants' characteristics according to MetS and TG levels. A statistically negligible difference in covariates was found in the following datasets: the matched, stratified and IPTW datasets for MetS in men; the matched and IPTW datasets for MetS in women; the matched, stratified and IPTW datasets for increased TG level in women.

The result of various analytical methods for the effect of MetS on CRC risk in men is summarized in Table 2. For the Cox's hazard regression models, the unadjusted and adjusted HRs between MetS and CRC were 0.96 (95\% confidence interval [Cl]: 0.51, 2.03) and 1.05 (95\% Cl: 0.54, 2.03). We considered that HRs estimated in any datasets except the matched, stratified, and IPTW datasets with imbalanced covariates were not reliable. The HRs from the PS 1:1 matched, stratified, and IPTW datasets were 0.92 (95\% Cl: 0.42, 2.04), 1.01 (95\% Cl: 0.53, 1.94), and 1.05 (95\% Cl: 0.72, 1.51), respectively, which were no significant.

Table 3 showed the associations of MetS and abnormal TG levels on CRC risk in women according to various analytical methods. The adjusted HRs in the unadjusted and multivariable Cox's hazard regression models were 2.37 (95\% Cl: 1.38, 4.06) and 2.11 (95\% Cl: 1.21, 3.68), respectively. We considered that HRs estimated in any datasets with imbalanced covariates were not reliable. The HR from the PS 1:1 matching analysis was $2.19(95 \% \mathrm{Cl}: 1.10,4.36)$ and that from the IPTW analysis was 2.02 (95\% Cl: 1.39, 2.93). Besides, the unadjusted and adjusted HRs between abnormal TG levels and CRC were 2.31 (95\% Cl: 1.35, 3.94) and 2.09 (95\% Cl: 1.21, 3.62), respectively. The HRs from the PS 1:1 matched, stratified, and IPTW datasets were 2.10 (95\% Cl: 1.08, 4.08), 2.22 (95\% Cl: 1.30, 3.78), and 2.01 
(95\% Cl: 1.38, 2.92), respectively, which were the only HRs estimated from datasets with balanced covariates.

\section{Discussion}

In this community-based cohort study in the Republic of Korea, we found that an increased risk of CRC in women associated with MetS and abnormal TG level by conducting PS-based analyses. In women, both MetS and abnormal TG level were associated with an approximately 2.0-fold to 2.2-fold increased risk of CRC. However, we showed that there was no significant association between MetS and CRC in men no matter which analytic methods we performed.

To our knowledge, our study is the first cohort study using PS-based methods to examine the effect of MetS on CRC incidence for both sexes in the Asian population. It seems that previous research findings were inconsistent: A meta-analysis reported that there was the significant association between MetS and CRC in both sexes in cohort studies across populations including the United States, European, Asian, and other populations (RR for men, 1.25 [95\% Cl, 1.19, 1.32]; RR for women, 1.34, [95\% Cl, 1.09, 1.64]) [2]. In another meta-analysis, the results in cohort studies across populations showed that men with MetS had a significantly elevated risk on CRC, but women with MetS did not [4]. However, in the United States, a cohort study that examined for postmenopausal women showed a similar risk level to ours (HR, 2.15; $95 \% \mathrm{Cl}, 1.30,3.53)$ [19]; the subsequent study recruited more participants but reported non-significant lower risks (HR, 1.16; 95\% Cl, 0.95-1.41) [20].

To discuss the association between MetS and CRC for the Asian population, two previous meta-analyses reported results using a couple of cohort studies for the Asian population: One showed that there was no association in cohort studies (RR for men, 1.10 [95\% Cl, 0.80, 1.51]; RR for women, 1.02, [95\% Cl, 0.76, 1.36]) [2]. Another also found that RRs for men and women from datasets across study designs were non-significant (RR for men, 1.23 [95\% Cl, 0.80, 1.88]; RR for women, 1.12, [95\% Cl, 0.86, 1.48]) [4]. In the case of the Republic of Korea, there were prior cohort studies about this association. Of these two studies using colon or colorectal adenoma risks as outcomes, one reported no association, but the other showed an association (adjusted HR, 1.28; 95\% Cl, 1.09-1.51) [6, 7]. The subjects of these cohort studies underwent colonoscopy for a health examination in a large hospital and could, therefore, be generally regarded as individuals who are interested in the prevention of future disease and the pursuit of a healthy lifestyle. Two studies using the National Health Insurance Service-National Sample Cohort to represent the Korean population reported somewhat conflicting results: One found only a significantly increased risk of colon cancer, not rectum cancer, for men with MetS (HR, $1.40[95 \% \mathrm{Cl}, 1.14,1.71])$, but did not find significant colon or rectum cancer risks for women [5]; the other found that MetS was associated with the development of CRC in both sexes (HR for men, 1.41 [95\% Cl, 1.37, 1.44]; HR for women, 1.23 [95\% Cl, $1.20,1.27]$ ] [21]. Our finding seems to be slightly different from previous studies above, as we have estimated using PS-based methods and study subjects of our study were just community residents. Thus, we expect that this study might contribute to providing additional evidence that there is the association 
between MetS and an elevated risk of CRC in the Asian population and future large cohort studies using PS-based methods could provide more definitive evidence.

In addition, our study showed that abnormal TG levels were associated with CRC risk in women. However, a previous review and meta-analysis reported that TG was not related to the risk of CRC [4]. Cohort studies in the United States and Japan also arrived at similar conclusions [19, 22]. In the Republic of Korea, previous cohort studies showed the HRs of colorectal adenoma to be $1.19(95 \% \mathrm{Cl}, 1.03,1.37)$ and $0.76(95 \% \mathrm{Cl}, 0.45,1.27)[6,7]$ and the HR of $\mathrm{CRC}$ to be $1.15(95 \% \mathrm{Cl}, 1.07,1.23)$ [21]. Future studies are recommended as it remains controversial whether the risk of CRC or colorectal adenoma is elevated when individuals' levels of TG are $150 \mathrm{mg} / \mathrm{dL}$ or higher.

As is well known, MetS is widely known as a risk factor for CRC, but the biological mechanism underlying this association remains to be clarified. Insulin resistance, systemic inflammation, and oxidative stress, and higher leptin levels have been suggested as potential mechanisms that may explain the association between MetS and CRC [3]. Insulin increases cell proliferation and reduces apoptosis, which may lead to tumor development. Insulin also induces the overstimulation of receptors of insulin-like growth factor-1 and 2 (IGF-1 and IGF-2), a key promoter of tumor development. Besides, deteriorated metabolic status influences elevated levels of inflammatory cytokines such as interleukin 6 (IL-6), tumor necrosis factor alpha (TNF-a), C-reactive protein (CRP), and leptin hormone, which may be implicated in insulin resistance and tumor development. Further studies are required to elucidate the mechanism underlying the effect of each component of MetS on CRC.

The strengths of this study include its prospective nature (i.e., cohort design), the strong causality between MetS and CRC by using PS-based methods. The selection biases that are present in most observational studies may lead to a lack of causality in this study. To improve the causality between MetS and CRC incidence, we performed PS-based analyses in a community-based cohort. A major advantage of using PS-based methods in observational studies can minimize selection biases by balancing nonrandomized individuals' data to reach the level of causality determined by randomized controlled trials. Recently, there have been some well-designed studies that have revealed associations between MetS and non-communicable diseases, including cancers, using PS-based analyses [23, 24].

There were several limitations to this study. We could not observe the following confounders: the history of MetS before cohort entry, individuals' stressful events, menopause, the consumption of carbohydrate and starchy foods, etc. Also, there is the possibility of information bias due to the use of a self-reported questionnaire. Also, we measured MetS only at the entry of this cohort study, so we could not estimate the risk of $\mathrm{CRC}$ due to changes in MetS over time. Furthermore, we used BMI as a measure of abdominal obesity of MetS due to the absence of WC data, although BMI and WC have slightly difference in the pathological meaning of MetS. Last, we could not obtain mortality data between 2014 and 2016 for noncancer cases among the study population from Statistics Korea, so we could not calculate a time-to-event outcome of performing survival analyses. 
In conclusion, this study may provide additional evidence that deteriorated metabolic profiles increase the risk of CRC in women. We highlight the importance of effective population-level interventions for deteriorated metabolic profiles at the early stages.

\section{List Of Abbreviations}

BMI: body mass index, Cl: confidence interval, CRC: colorectal cancer, DBP: diastolic blood pressure, FBS: fasting blood sugar, HDL: high-density lipoprotein, HR: hazard risk, IPTW: inverse probability-of-treatment weighted, MetS: metabolic syndrome, PS: propensity score, SBP: systolic blood pressure, SMRW: standardized mortality ratio weighted, TG: triglyceride, WC: waist circumference

\section{Declarations}

\section{Ethics approval and consent to participate}

The study was approved by the KNCC Institutional Review Board (IRB No. NCC2016-0300). All participants had provided written informed consent.

\section{Consent for publication}

Not applicable

\section{Availability of data and materials}

Not applicable

\section{Competing interests}

The authors declare no potential conflicts of interest.

\section{Funding}

This work was supported by a research grant from the National Cancer Center, the Republic of Korea (No. 1710240-3). The funders had no role in study design, data collection and analysis, decision to publish, or preparation of the manuscript.

\section{Authors' contributions}

EYP contributed to conceptualization and methodology of this study and helped in writing the manuscript. JK drafted the manuscript and performed the statistical analysis. EP and BK contributed to the statistical analysis and interpretation of the results. MKL involved in the design and coordination of the study. JKO involved in data collection in the field and questionnaire design.

\section{Acknowledgements}


The authors appreciate all the collaborators, including those at the public health centers of Haman-gun, Changwon-si, Chungju-si and Chuncheon-si, and the Health Center and County Hospital of Sancheonggun, for their support with the implementation of the field surveys, clinical laboratory examinations and data collection, retrieval and analyses.

\section{Author's information}

J.S. Kim has moved to the Division of TB and HIV / AIDS Control, Center for Disease Prevention, and Korea Centers for Disease Control \& Prevention during the research. Division of TB and HIV / AIDS Control, Center for Disease Prevention, and Korea Centers for Disease Control \& Prevention, (28159) Osong Health Technology Administration Complex, 187, Osongsaengmyeong 2-ro, Osong-eup, Heungdeok-gu, Cheongju-si, Chungcheongbuk-do, Korea.

\section{References}

1. Bray F, Ferlay J, Soerjomataram I, Siegel RL, Torre LA, Jemal A. Global cancer statistics 2018 : GLOBOCAN estimates of incidence and mortality worldwide for 36 cancers in 185 countries. CA Cancer J Clin. 2018;68:394-424.

2. Esposito K, Chiodini P, Colao A, Lenzi A, Giugliano D. Metabolic syndrome and risk of cancer: a systematic review and meta-analysis. Diabetes Care. 2012;35:2402-11.

3. Mendonca FM, de Sousa FR, Barbosa AL, Martins SC, Araujo RL, Soares R, et al. Metabolic syndrome and risk of cancer: which link? Metabolism. 2015;64:182-9.

4. Esposito K, Chiodini P, Capuano A, Bellastella G, Maiorino MI, Rafaniello C, et al. Colorectal cancer association with metabolic syndrome and its components: a systematic review with meta-analysis. Endocrine. 2013;44:634-47.

5. Ko S, Yoon SJ, Kim D, Kim AR, Kim EJ, Seo HY. Metabolic risk profile and cancer in Korean men and women. J Prev Med Public Health. 2016;49:143-52.

6. Kim NH, Park JH, Park DI, Sohn Cl, Choi K, Jung YS. Metabolic syndrome is a risk factor for adenoma occurrence at surveillance colonoscopy: a single-center experience in Korea. Medicine (Baltimore). 2016;95:e4454.

7. Kim MC, Kim CS, Chung TH, Park HO, Yoo Cl. Metabolic syndrome, lifestyle risk factors, and distal colon adenoma: a retrospective cohort study. World J Gastroenterol. 2011;17:4031-7.

8. Haukoos JS, Lewis RJ. The propensity score. JAMA. 2015;314:1637-8.

9. Austin PC. An introduction to propensity score methods for reducing the effects of confounding in observational studies. Multivariate Behav Res. 2011;46:399-424.

10. Tak YJ, Lee SY, Park SK, Kim YJ, Lee JG, Jeong DW, et al. Association between uterine leiomyoma and metabolic syndrome in parous premenopausal women: a case-control study. Medicine (Baltimore). 2016;95:e5325. 
11. Won KB, Chang HJ, Han D, Sung J, Choi SY. Metabolic syndrome predicts long-term mortality in subjects without established diabetes mellitus in asymptomatic Korean population: a propensity score matching analysis from the Korea Initiatives on Coronary Artery Calcification (KOICA) registry. Medicine (Baltimore). 2016;95:e5421.

12. Oh JK, Lim MK, Yun EH, Choi MH, Hong ST, Chang SH, et al. Cohort profile: community-based prospective cohort from the national cancer center, Korea. Int J Epidemiol. 2017;46:e14.

13. Grundy SM, Cleeman JI, Merz CN, Brewer HB, Jr., Clark LT, Hunninghake DB, et al. Implications of recent clinical trials for the national cholesterol education program adult treatment panel III guidelines. Circulation. 2004;110:227-39.

14. Ahmed RL, Schmitz KH, Anderson KE, Rosamond WD, Folsom AR. The metabolic syndrome and risk of incident colorectal cancer. Cancer. 2006;107:28-36.

15. Jinjuvadia R, Lohia P, Jinjuvadia C, Montoya S, Liangpunsakul S. The association between metabolic syndrome and colorectal neoplasm: systemic review and meta-analysis. J Clin Gastroenterol. 2013;47:33-44.

16. Desai RJ, Rothman KJ, Bateman BT, Hernandez-Diaz S, Huybrechts KF. A propensity-score-based fine stratification approach for confounding adjustment when exposure is infrequent. Epidemiology. 2017;28:249-57.

17. Kurth T, Walker AM, Glynn RJ, Chan KA, Gaziano JM, Berger K, et al. Results of multivariable logistic regression, propensity matching, propensity adjustment, and propensity-based weighting under conditions of nonuniform effect. Am J Epidemiol. 2006;163:262-70.

18. Yoshida K, Bohn J. Package 'tableone' for R statistical programming language. 2018. https://cran.rproject.org/web/packages/tableone/tableone.pdf. Accessed 4 Apr 2018.

19. Kabat GC, Kim MY, Peters U, Stefanick M, Hou L, Wactawski-Wende J, et al. A longitudinal study of the metabolic syndrome and risk of colorectal cancer in postmenopausal women. Eur J Cancer Prev. 2012;21:326-32.

20. Kabat GC, Kim MY, Stefanick M, Ho GYF, Lane DS, Odegaard AO, et al. Metabolic obesity phenotypes and risk of colorectal cancer in postmenopausal women. Int J Cancer. 2018;143:543-51.

21. Choi YJ, Lee DH, Han KD, Shin CM, Kim N. Abdominal obesity, glucose intolerance and decreased high-density lipoprotein cholesterol as components of the metabolic syndrome are associated with the development of colorectal cancer. Eur J Epidemiol. 2018;33:1077-85.

22. Inoue M, Noda M, Kurahashi N, Iwasaki M, Sasazuki S, Iso H, et al. Impact of metabolic factors on subsequent cancer risk: results from a large-scale population-based cohort study in Japan. Eur $\mathrm{J}$ Cancer Prev. 2009;18:240-7.

23. Ahmadi A, Leipsic J, Feuchtner G, Gransar H, Kalra D, Heo R, et al. Is metabolic syndrome predictive of prevalence, extent, and risk of coronary artery disease beyond its components? Results from the multinational coronary CT angiography evaluation for clinical outcome: an international multicenter registry (CONFIRM). PLoS One. 2015;10:e0118998. 
24. Kheterpal E, Sammon JD, Diaz M, Bhandari A, Trinh QD, Pokala N, et al. Effect of metabolic syndrome on pathologic features of prostate cancer. Urol Oncol. 2013;31:1054-9.

\section{Tables}

\section{Table 1. Baseline Characteristics of Study Participants}

\begin{tabular}{|c|c|c|c|c|c|}
\hline \multirow[t]{3}{*}{ Characteristics } & \multicolumn{3}{|c|}{ Male } & \multicolumn{2}{|l|}{ Female } \\
\hline & \multicolumn{2}{|c|}{ Colorectal cancer } & $P$-value & Colorectal cancer & $P$-value \\
\hline & $\overline{\text { No }(N=2,360)}$ & Yes $(\mathrm{N}=57)$ & & No $(N=4,514)$ Yes $(N=54)$ & \\
\hline Age (years, Mean \pm SD) & $59.66 \pm 10.93$ & $64.18 \pm 8.67$ & 70.002 & $59.95 \pm 11.2 \quad 64.72 \pm 9.19$ & 90.001 \\
\hline Physical activity (days/week, Mean \pm SD) & $3.97 \pm 2.86$ & $4.26 \pm 2.7$ & 0.754 & $2.99 \pm 2.96$ & 0.6 \\
\hline Intake of fruits or vegetables (days/week, Mean \pm SD) & $5.43 \pm 1.44$ & $5.19 \pm 1.57$ & 0.28 & $5.51 \pm 1.46$ & 0.073 \\
\hline Intake of beef or pork (days/week, Mean \pm SD) & $2.55 \pm 1.4$ & $2.44 \pm 1.49$ & 0.732 & $1.31 \pm 1.33$ & 0.012 \\
\hline \multicolumn{6}{|l|}{ Alcohol consumption $[\mathrm{N}(\%)]$} \\
\hline Non-drinkers & $640(27.12)$ & $7(12.28)$ & 0.03 & 3,598 (79.71) 45 (83.33) & 0.796 \\
\hline Moderate drinkers (<24 g/day) & $797(33.77)$ & $26(45.61)$ & & $797(17.66) \quad 8(14.81)$ & \\
\hline Heavy drinkers ( $\geq 24 \mathrm{~g} /$ day) & $923(39.11)$ & $24(42.11)$ & & $119(2.64)$ & \\
\hline \multicolumn{6}{|l|}{ Smoking status $[\mathrm{N}(\%)]$} \\
\hline Non-smokers & $483(20.47)$ & $12(21.05)$ & 0.952 & 4,186 (92.73) 52 (96.3) & 0.894 \\
\hline Moderate smokers (<20 pack-year) & $669(28.35)$ & $17(29.82)$ & & $263(5.83) \quad 2(3.7)$ & \\
\hline Heavy smokers ( $\geq 20$ pack-year) & $1,208(51.19)$ & $28(49.12)$ & & $65(1.44)$ & \\
\hline \multicolumn{6}{|l|}{ Education level [N(\%)] } \\
\hline Illiterate & $222(9.41)$ & $6(10.53)$ & 0.448 & $1,445(32.01) 24(44.44)$ & 0.24 \\
\hline Middle school or less & $1,443(61.14)$ & $39(68.42)$ & & $2,505(55.49) 26(48.15)$ & \\
\hline High school & $484(20.51)$ & $10(17.54)$ & & $438(9.7) \quad 3(5.56)$ & \\
\hline College or more & $211(8.94)$ & $2(3.51)$ & & $126(2.79)$ & \\
\hline \multicolumn{6}{|l|}{ Residential area [N(\%)] } \\
\hline Sancheong-gun & $1,270(53.81)$ & $23(40.35)$ & 0.209 & $2,380(52.72) 21(38.89)$ & 0.072 \\
\hline Changwon-si & $485(20.55)$ & $16(28.07)$ & & $867(19.21) \quad 13(24.07)$ & \\
\hline Chooncheon-si & $167(7.08)$ & $7(12.28)$ & & $438(9.7) \quad 3(5.56)$ & \\
\hline Choongjoo-si & $281(11.91)$ & $8(14.04)$ & & $558(12.36)$ & \\
\hline Haman-gun & $157(6.65)$ & $3(5.26)$ & & $6(11.11)$ & \\
\hline \multicolumn{6}{|l|}{ Metabolic syndrome $[\mathrm{N}(\%)]$} \\
\hline No (No. of components of MetS <3) & $1,848(78.31)$ & $45(78.95)$ & 0.907 & $3,243(71.84) 28(51.85)$ & 0.001 \\
\hline Yes (No. of components of MetS $\geq 3$ ) & $512(21.69)$ & $12(21.05)$ & & $1,271(28.16) 26(48.15)$ & \\
\hline \multicolumn{6}{|l|}{ Blood pressure $[\mathrm{N}(\%)]$} \\
\hline $\mathrm{SBP}<130 \mathrm{mmHg}$ and $\mathrm{DBP}<85 \mathrm{mmHg}$ & $996(42.2)$ & $21(36.84)$ & 0.418 & $2,017(44.68) 19(35.19)$ & 0.163 \\
\hline $\mathrm{SBP} \geq 130 \mathrm{mmHg}$ or $\mathrm{DBP} \geq 85 \mathrm{mmHg}$ & $1,364(57.8)$ & $36(63.16)$ & & $2,497(55.32) 35(64.81)$ & \\
\hline \multicolumn{6}{|l|}{ BMI $[N(\%)]$} \\
\hline$<25 \mathrm{~kg} / \mathrm{m}^{2}$ & $1,667(70.64)$ & $40(70.18)$ & 0.94 & $2,840(62.92) 33(61.11)$ & 0.785 \\
\hline$\geq 25 \mathrm{~kg} / \mathrm{m}^{2}$ & $693(29.36)$ & $17(29.82)$ & & $1,674(37.08) 21(38.89)$ & \\
\hline \multicolumn{6}{|l|}{ HDL cholesterol [N(\%)] } \\
\hline$\geq 50 \mathrm{mg} / \mathrm{dL}$ & $1,913(81.06)$ & $54(94.74)$ & 0.009 & $2,309(51.15) 26(48.15)$ & 0.661 \\
\hline$<50 \mathrm{mg} / \mathrm{dL}$ & $447(18.94)$ & $3(5.26)$ & & $2,205(48.85) 28(51.85)$ & \\
\hline \multicolumn{6}{|l|}{ Triglyceride level [N(\%)] } \\
\hline$<150 \mathrm{mg} / \mathrm{dL}$ & $1,552(65.76)$ & $40(70.18)$ & 0.488 & $3,148(69.74)$ & 0.002 \\
\hline$\geq 150 \mathrm{mg} / \mathrm{dL}$ & $808(34.24)$ & $17(29.82)$ & & $1,366(30.26)$ & \\
\hline \multicolumn{6}{|l|}{ FBS [N(\%)] } \\
\hline$<110 \mathrm{mg} / \mathrm{dL}$ & $2,047(86.74)$ & $46(80.7)$ & 0.186 & $4,042(89.54) 49(90.74)$ & 0.775 \\
\hline$\geq 110 \mathrm{mg} / \mathrm{dL}$ & $313(13.26)$ & $11(19.3)$ & & $472(10.46) \quad 5(9.26)$ & \\
\hline
\end{tabular}


SD, standard deviation; SBP, systolic blood pressure; DBP, diastolic blood pressure; BMI, body mass index; HDL, high-density lipoproteins; FBS, fasting blood sugar.

Table 2. Association between Metabolic Syndrome and Colorectal Cancer Incidence in Men

\begin{tabular}{lcccc}
\hline Methods & Cases $(\mathbf{N})$ & Controls (N) & HR (95\% CI) & $P$-value \\
\hline Cox's hazard regression & & & & \\
\hline$\quad$ Unadjusted & 57 & 2,360 & $0.96(0.51,1.83)$ & 0.908 \\
\hline$\quad$ Multivariable ${ }^{\text {a) }}$ & 57 & 2,360 & $1.05(0.54,2.03)$ & 0.892 \\
\hline $\begin{array}{l}\text { PS-based Cox's hazard regression } \\
\quad \text { Matched for PS }\end{array}$ & 25 & 1,013 & $0.92(0.42,2.04)$ & 0.84 \\
\hline$\quad$ Stratification into 5 strata by PS & 57 & 2,345 & $1.01(0.53,1.94)$ & 0.968 \\
\hline$\quad$ Regression adjusted with PS & & & & \\
$\quad$ as a continuous term & 57 & 2,360 & $1.05(0.54,2.02)$ & 0.893 \\
\hline$\quad$ as a quintile term & 57 & 2,360 & $1.02(0.53,1.96)$ & 0.956 \\
\hline$\quad$ Weighted models & & & & \\
$\quad$ IPTW model & 57 & 2,360 & $1.05(0.72,1.51)$ & 0.81 \\
\hline$\quad$ SMRW model & 57 & 2,360 & $0.95(0.53,1.71)$ & 0.873 \\
\hline$\quad$
\end{tabular}

HR, hazard ratio; CI, confidence interval; PS, propensity score; IPTW, inverse probability-of-treatment weighted; SMRW, standardized mortality ratio weighted. a)Adjusted by age, education, smoking status, alcohol consumption, physical activity, frequency of intake of fruits or vegetables, frequency of intake of red meats, and residential area.

Table 3. Association between (a) Metabolic Syndrome, (b) Triglyceride Level and Colorectal Cancer Incidence in Women.

\begin{tabular}{|c|c|c|c|c|}
\hline Methods & Cases (N) & Controls (N) & HR (95\% CI) & $P$-value \\
\hline \multicolumn{5}{|l|}{ (a) Metabolic syndrome } \\
\hline \multicolumn{5}{|l|}{ General Cox's hazard regression } \\
\hline Unadjusted & 54 & 4,514 & $2.37(1.38,4.06)$ & 0.002 \\
\hline Multivariable $^{\text {a) }}$ & 54 & 4,514 & $2.11(1.21,3.68)$ & 0.009 \\
\hline \multicolumn{5}{|l|}{ PS-based Cox's hazard regression } \\
\hline Matched for PS & 38 & 2,542 & $2.19(1.10,4.36)$ & 0.026 \\
\hline Stratification into 5 strata by PS & 53 & 4,505 & $2.19(1.29,3.72)$ & 0.004 \\
\hline \multicolumn{5}{|l|}{ Regression adjusted with PS } \\
\hline as a continuous term & 54 & 4,514 & $2.03(1.17,3.53)$ & 0.012 \\
\hline as a quintile term & 54 & 4,514 & $2.07(1.19,3.59)$ & 0.01 \\
\hline \multicolumn{5}{|l|}{ Weighted models } \\
\hline IPTW model & 54 & 4,514 & $2.02(1.39,2.93)$ & $<0.001$ \\
\hline SMRW model & 54 & 4,514 & $2.52(1.66,3.84)$ & $<0.001$ \\
\hline \multicolumn{5}{|l|}{ (b) Triglyceride level } \\
\hline \multicolumn{5}{|l|}{ General Cox's hazard regression } \\
\hline Unadjusted & 54 & 4,514 & $2.31(1.35,3.94)$ & 0.002 \\
\hline Multivariable ${ }^{a)}$ & 54 & 4,514 & $2.09(1.21,3.62)$ & 0.008 \\
\hline \multicolumn{5}{|l|}{ PS-based Cox's hazard regression } \\
\hline Matched for PS & 40 & 2,746 & $2.10(1.08,4.08)$ & 0.029 \\
\hline Stratification into 5 strata by PS & 53 & 4,501 & $2.22(1.30,3.78)$ & 0.003 \\
\hline \multicolumn{5}{|l|}{ Regression adjusted with PS } \\
\hline as a continuous term & 54 & 4,514 & $2.06(1.19,3.55)$ & 0.01 \\
\hline as a quintile term & 54 & 4,514 & $2.07(1.20,3.57)$ & 0.009 \\
\hline \multicolumn{5}{|l|}{ Weighted models } \\
\hline IPTW model & 54 & 4,514 & $2.01(1.38,2.92)$ & $<0.001$ \\
\hline SMRW model & 54 & 4,514 & $2.46(1.62,3.74)$ & $<0.001$ \\
\hline
\end{tabular}


HR, hazard ratio; CI, confidence interval; PS, propensity score; IPTW, inverse probability-of-treatment weighted; SMRW, standardized mortality ratio weighted. ${ }^{a}$ Adjusted by age, education, smoking status, alcohol consumption, physical activity, frequency of intake of fruits or vegetables, frequency of intake of red meats, and residential area.

\section{Figures}




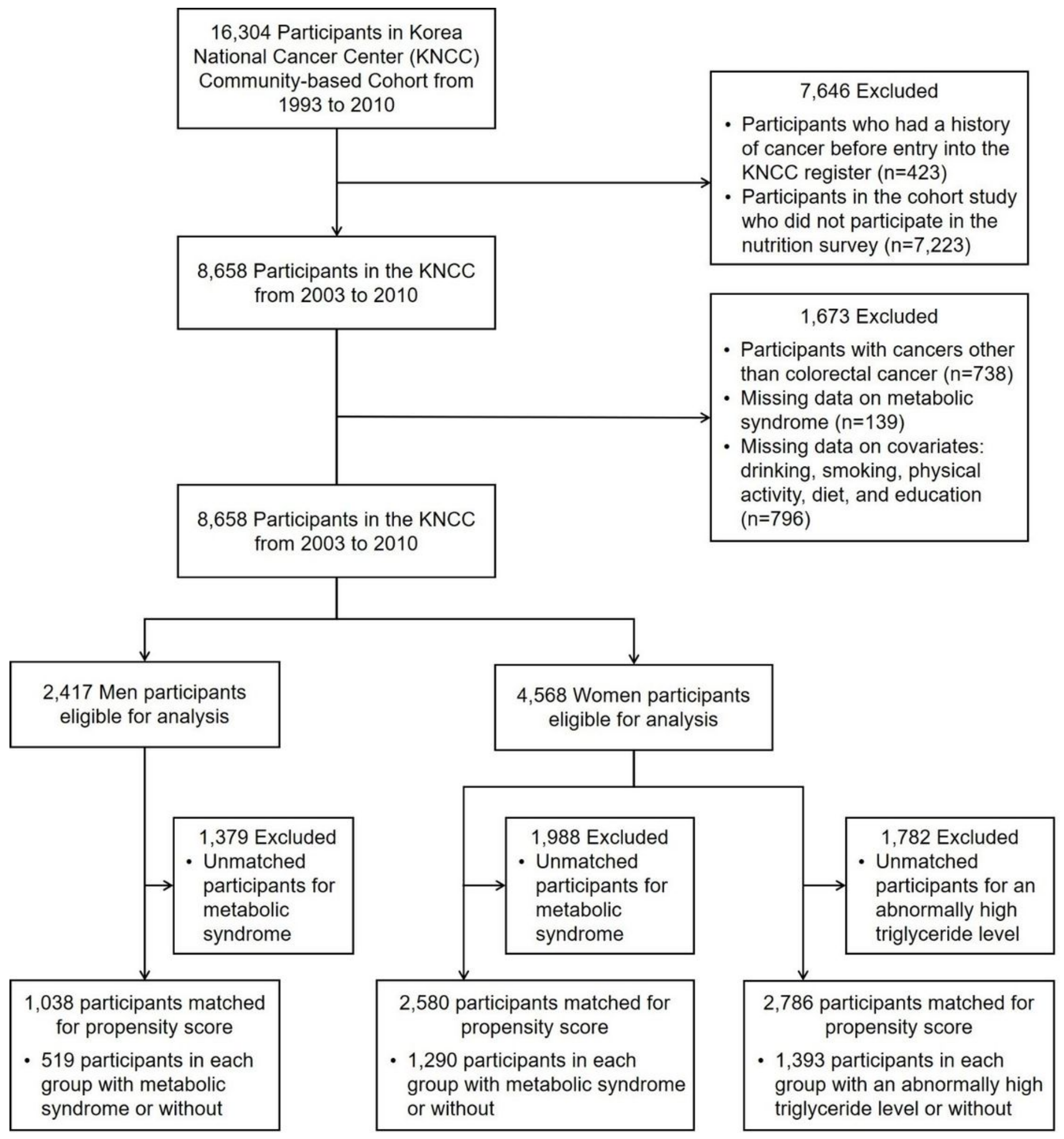

\section{Figure 1}

Flow Diagram of the Derivation of the Study Population.

\section{Supplementary Files}

This is a list of supplementary files associated with this preprint. Click to download. 
- SupplementaryFigureS1.jpg

- SupplementaryFigureS2.jpg 